\title{
Smith-Purcell radiation from ultraviolet to infrared using a Si field emitter
}

\author{
Yoichro $\mathrm{Neo}^{\mathrm{a})}$ \\ Research Institute of Electronics, Shizuoka University, 3-5-1 Johoku, Hamamatsu 432-8011, Japan \\ Hidetaka Shimawaki \\ Hachinohe Institute of Technology, Ohbiraki, Myo, Hachinohe 031-8501, Japan \\ Takahiro Matsumoto \\ Research and Development Center, Stanley Electric Corporation, 1-3-1 Eda-nishi, Aoba-ku, Yokohama, \\ Kanagawa 225-0014, Japan \\ Hidenori Mimura \\ The Graduate School of Electronic Science and Technology, Research Institute of Electronics, Shizuoka \\ University, 3-5-1 Johoku, Hamamatsu 432-8011, Japan
}

(Received 2 September 2005; accepted 17 January 2006; published 27 March 2006)

\begin{abstract}
The first, second, third, and fourth harmonics of Smith-Purcell radiation in a broadband ranging from $330 \mathrm{~nm}$ in the ultraviolet range to $1350 \mathrm{~nm}$ in the near infrared range have been detected using a $416 \mathrm{~nm}$ pitch grating. We used a single tip Si field emitter as an electron source and the input power level was low such as beam currents of 20-200 nA and acceleration voltages of 25-34 kV. Emission peaks in the Smith-Purcell radiation blueshift with increasing the acceleration voltage and coincide with the theoretical ones. () 2006 American Vacuum Society. [DOI: 10.1116/1.2174023]
\end{abstract}

\section{INTRODUCTION}

Smith-Purcell radiation (SPR) is generated by the interaction between traveling charged particles and a periodic metallic structure. SPR covers broad wide range from visible light to millimeter waves. ${ }^{1-4}$ The radiated wavelength of SPR is simply expressed by

$$
\lambda=d(1-\beta \cos \theta) / \beta n,
$$

where $\lambda$ is the wavelength, $d$ is the grating period, $\theta$ is the emission angle $\left(0^{\circ}\right.$ refers to the parallel to the electron beam), $n$ is the harmonic number, and $\beta$ is the ratio of electron velocity to light one. Recently, SPR has been recognized as a promising light source from visible light to far infrared, especially in far infrared [terahertz in the frequency range], because a compact, continuous, and tunable light source is not available in the tetrahertz region. Our final goal in the research of SPR is the development of an efficient and miniature-size free electron laser in the terahertz region by using a field emitter array as an electron source. In the previous report, we fabricated a SPR system using a single tip Si field emitter (FE) and successfully observed the SPR radiation in the visible range at a low input power level with beam currents of 20-200 nA and acceleration voltages of $25-30 \mathrm{kV}$. 5 The reason why we used such a low input power level and a semiconductor field emitter is because we aim to develop a miniature-size SPR free electron laser and the electron beam from a semiconductor one is relatively easily modulated by laser irradiation, leading to the formation of a bunched beam (short pulse beam train), respectively. ${ }^{6}$ The bunched beam effectively enhances SPR due to the coherent effect. $^{7}$

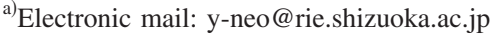

In this article, besides the visible SPR we tried to detect SPR in ultraviolet and near infrared regions by using the SRR system with a single tip Si field emitter. As a result, we have detected SPR in the ultraviolet and near infrared regions.

\section{EXPERIMENTS}

A single tip Si FE was used as an electron source in the SPR experiment. A conical shaped Si FE was fabricated by a conventional method using reactive ion etching (RIE) and thermal oxidation sharpening. An $n$-type (100)-oriented single-crystal $\mathrm{Si}$ wafer with a resistivity of $2-3 \Omega \mathrm{cm}$ was used as an emitter substrate. The emitter has a $1.5 \mu \mathrm{m}$ diam gate aperture and a $1 \mu \mathrm{m}$ tip height. The SPR system is shown in Fig. 1. All SPR experiments were carried out in an ultrahigh vacuum chamber, which was pumped below 1.5 $\times 10^{-7} \mathrm{~Pa}$. The field emitter was set on a cathode shank, located in the center of the chamber and biased at about $-30 \mathrm{kV}$. The cathode shank has a wehnelt geometry, which was designed for electron beam sharpening using a simulator. Electron beam must skim a grating, because the electric field interacting with the electron beam decreases exponentially with increasing the beam height from the grating surface. An aluminum-coated replica grating with $2.5 \mathrm{~cm}$ wide and $2.5 \mathrm{~cm}$ long was located $3 \mathrm{~cm}$ in front of the cathode so that electron beam from the cathode passed over the grating surface. The anode consisting of two parts, upper and lower plates separated by a $0.5 \mathrm{~mm}$ wide aperture, was set on the entrance side of the grating. The both plates also work as a deflector by applying a bias voltage of $\pm 500 \mathrm{~V}$ on each plate. The gate voltage applied to the Si FE was around $80 \mathrm{~V}$ in the experiments. Though the emission current at the gate voltage of $80 \mathrm{~V}$ was about a few microamperes, the deflectors cut over $90 \%$ of the emission current. Consequently, the real 


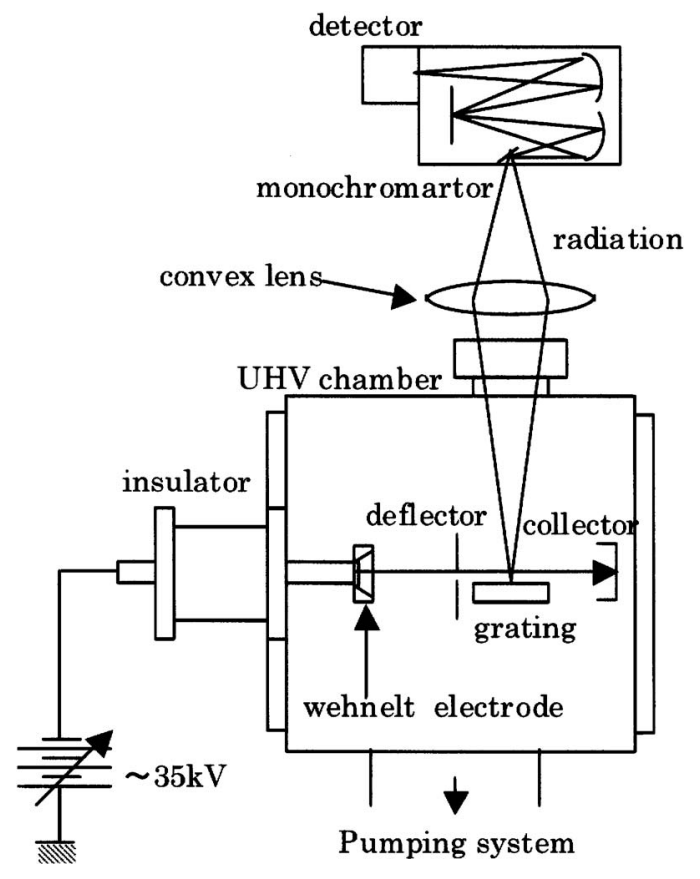

FIG. 1. Schematic setup of SPR experimental apparatus. Liquid N2 cooled $\mathrm{CCD}$ and InGaAs detectors were used for ultraviolet-visible and infrared regions, respectively.

traveling electron beam was 20-200 nA. SPR was detected through a viewing port. We collected SPR with an emission angle between $80^{\circ}$ and $110^{\circ}$ using a convex lens. liquid $\mathrm{N}_{2}$ cooled charge-coupled device (CCD) and InGaAs detectors were used for ultraviolet-visible and near infrared regions, respectively.

Figure 2 shows the calculation of the relation between the acceleration voltage and the grating pitch for various har-

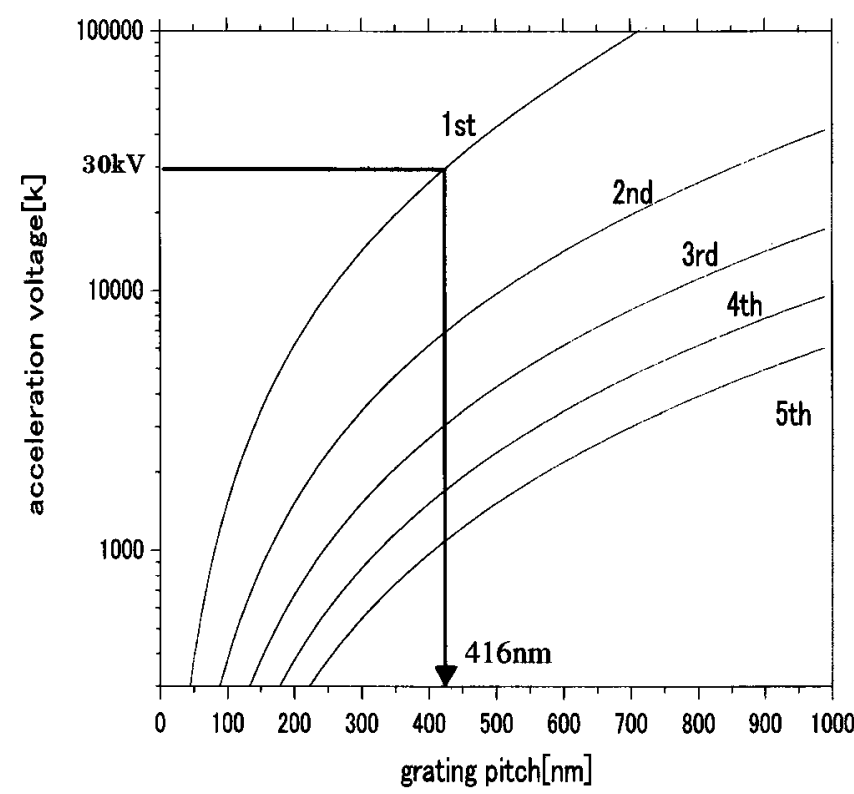

FIG. 2. Calculation of the relation between the acceleration voltage and the grating pitch for various harmonic numbers. The SP emission angle of $90^{\circ}$ and the wavelength of $1.3 \mu \mathrm{m}$ were used in the calculation.

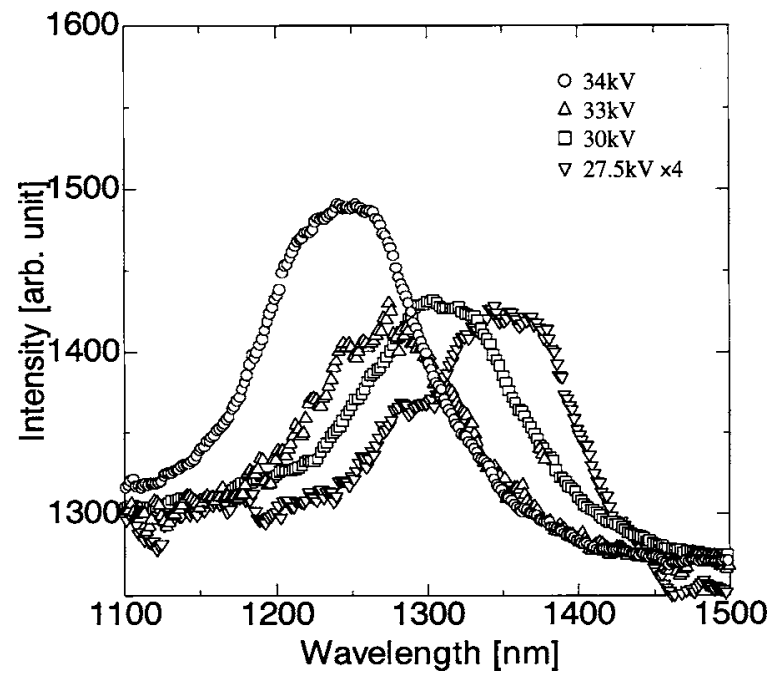

FIG. 3. SPR spectra in the near infrared region for the acceleration voltage of $27.5,30,33$, and $34 \mathrm{kV}$. The spectrum at $27.5 \mathrm{kV}$ was magnified four times.

monic numbers. In this calculation, we set the SP emission angle of $90^{\circ}$ and the wavelength of $1.3 \mu \mathrm{m}$. The calculation indicates that when a grating with a $416 \mathrm{~nm}$ pitch is used, SPR with a peak wavelength of $1.3 \mu \mathrm{m}$ is obtained for the acceleration voltage of $30 \mathrm{kV}$ and first harmonic number. Therefore, we used a $416 \mathrm{~nm}$ pitch grating.

\section{RESULTS AND DISCUSSION}

Figure 3 shows the SPR spectra in the near infrared region. As calculated in Fig. 2, a peak is clearly observed at $1.3 \mu \mathrm{m}$ for the acceleration voltage of $30 \mathrm{kV}$. The peak blueshifts with increasing the acceleration voltage from 27.5 to $34 \mathrm{kV}$. The spectrum of $27.5 \mathrm{kV}$ was magnified four times. These peaks were considered as the first harmonics of the SPR.

Figure 4 shows the SPR spectra in the ultraviolet and visible regions for the acceleration voltage from 25 to $30 \mathrm{kV}$. The insert shows the enlarged spectra from 250 to $400 \mathrm{~nm}$. Three groups of peaks around 350, 400, and $600 \mathrm{~nm}$ correspond to the fourth, third, and second harmonics of the SPR, respectively. These peaks blueshift with increasing the acceleration voltage. On the other hand, small peaks around 450 and $500 \mathrm{~nm}$ probably come from the soda glass, which is used as a substrate of the grating. The SPR with a shorter wavelength than $300 \mathrm{~nm}$ is not detected because of the opaque characteristic of the glass used in the viewing port.

Figure 5 shows the dependence of the SPR wavelength on the acceleration voltage for the emission angle of $90^{\circ}$. The solid lines and circles are the calculated and measured results, respectively. The measurement peaks almost coincide with the calculated ones, although there is a small discrepancy between them. The reason of the small discrepancy is that the center of the detection angle in the experiment is not just $90^{\circ}$. The first, second, third, and fourth harmonics of SPR are observed in the wide range from ultraviolet to near infrared. SPR intensity could be calculated by using theoret- 


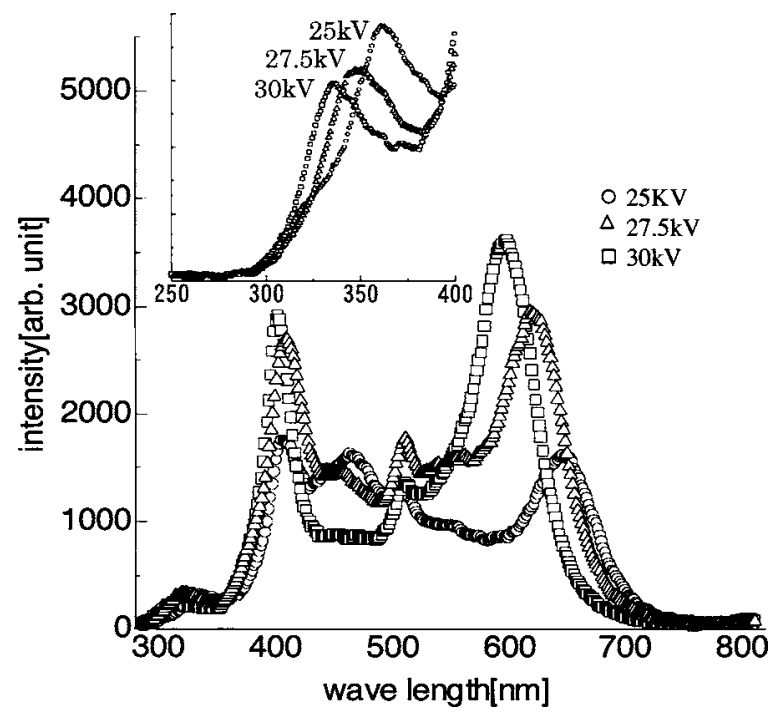

FIG. 4. SPR spectra in ultraviolet and visible regions for the acceleration voltage of $25,27.5$, and $30 \mathrm{kV}$. The insert shows the enlarged spectra from 250 to $400 \mathrm{~nm}$.

ical formula. ${ }^{8}$ Even with the optimized electro-optics conditions, the estimated SPR intensity could not achieve to $10^{-15} \mathrm{~W} / \mathrm{sr}$ with our input conditions. In order to increase SPR intensity for the practical application, the short pulse beams and the cavity structure, such as Fabry-Pérot oscillator, should be induced in our system.

Figure 6 shows the summary of the peak wavelengths of the SPR detected by our system. The figure includes the SPR peaks detected with a $550 \mathrm{~nm}$ pitch grating. ${ }^{5} \mathrm{SPR}$ in the wide range from 330 to $1350 \mathrm{~nm}$ is clearly observed and the measured wavelengths coincide with the predicted ones form the SPR theory.

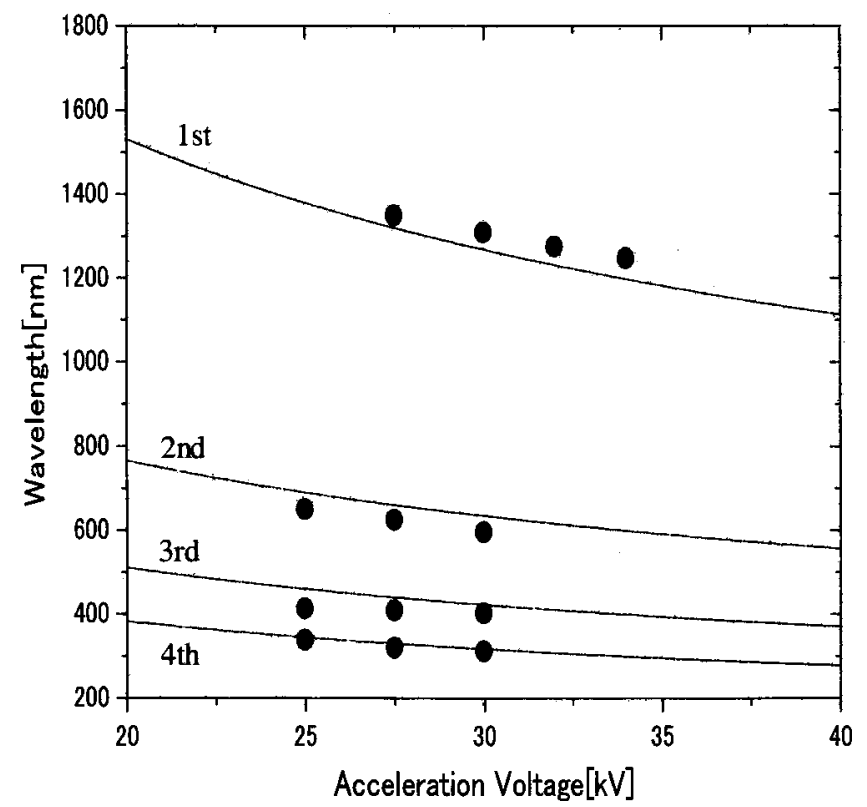

FIG. 5. Dependence of the SPR wavelength on the acceleration voltage for the emission angle of $90^{\circ}$. The solid lines and circles are the calculated and measured results, respectively.

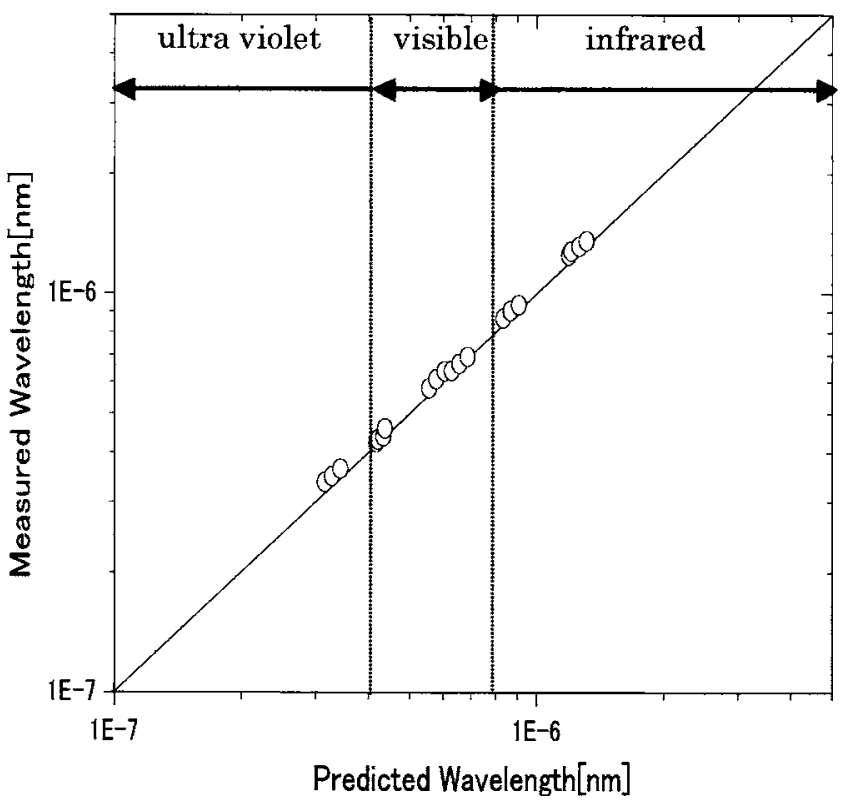

FIG. 6. Summary of the peak wavelengths of the SPR detected by our system. The measured wavelengths coincide with the predicted ones form the SPR theory.

\section{SUMMARY}

We have fabricated a compact SPR system using a single tip Si field emitter with a conical shape and an aluminumcoated $2.5 \mathrm{~cm}$ wide and $2.5 \mathrm{~cm}$ long replica grating. Using a $416 \mathrm{~nm}$ pitch grating, we have detected the first, second, third, and fourth harmonics of SPR in a broadband ranging from $330 \mathrm{~nm}$ in the ultraviolet range to $1350 \mathrm{~nm}$ in the near infrared range at the low input level such as beam currents of 20-200 nA and acceleration voltages of $25-34 \mathrm{kV}$. These results indicate that the SPR system using a semiconductor field emitter is a promising light source.

\section{ACKNOWLEDGMENTS}

This work was partially supported by Grant-in-Aids for Scientific Research from the Ministry of Education, Science, Sports, and Culture of Japan and for Top Priority R\&D Home Affairs, Posts, and Telecommunications.

${ }^{1}$ C. M. Tang, M. Goldstein, T. A. Swyden, and J. E. Walsh, Nucl. Instrum. Methods Phys. Res. A 358, 214 (1995).

${ }^{2}$ H. Ishizuka, Y. Kawamura, K. Yokoo, H. Shimawaki, and A. Hosono, Nucl. Instrum. Methods Phys. Res. A 445, 276 (2000).

${ }^{3}$ H. Ishizuka, Y. Kawamura, K. Yokoo, H. Shimawaki, and A. Hosono, Nucl. Instrum. Methods Phys. Res. A 475, 593 (2001).

${ }^{4}$ H. Mimura, H. Ishizuka, and K. Yokoo, Nucl. Instrum. Methods Phys. Res. A 528, 497 (2004).

${ }^{5}$ Y. Neo, Y. Suzuki, K. Sagae, H. Shimawaki, and H. Mimura, J. Vac. Sci. Technol. B 23, 840 (2005).

${ }^{6}$ H. Mimura, T. Ukeba, H. Shimawaki, and K. Yokoo, J. Vac. Sci. Technol. B 22, 1218 (2004).

${ }^{7}$ J. E. Walsh, J. Brownell, J. C. Swartz, J. Urata, and M. F. Kimmitt, Nucl. Instrum. Methods Phys. Res. A 429, 457 (1999).

${ }^{8}$ J. E. Wash, Nucl. Instrum. Methods Phys. Res. A 445, 214 (2000). 